\title{
Polarity-switching Top Coats for Silicon-containing Block Copolymer Orientation Control
}

\author{
Christopher M. Bates ${ }^{1}$, Michael J. Maher ${ }^{1}$, Anthony Thio ${ }^{2}$, Leon M. Dean ${ }^{2}$, Julia D. Cushen ${ }^{2}$, William \\ J. Durand ${ }^{2}$, Greg Blachut ${ }^{2}$, Litan Li $^{2}$, Christopher J. Ellison ${ }^{2}$ and C. Grant Willson ${ }^{1,2}$
}

The University of Texas at Austin, Department of Chemistry ${ }^{1}$, Department of Chemical Engineering, 200 E. Dean Keeton Stop C0400 Austin, TX 78712

Keywords: block copolymer, top coat, thermal annealing, directed self-assembly

\section{Introduction}

Block copolymers (BCPs) are a class of soft materials that can self-assemble into ordered nano-structures circa 3-100 $\mathrm{nm} .{ }^{1}$ The lower range of accessible length scales surpasses sub-193 immersion lithography resolution limits and thus provides great promise for next-generation lithographic technologies. Effectively harnessing the capabilities of BCPs in thin films requires simultaneous control of morphology, domain orientation, and alignment (i.e. long-range order). $\mathrm{BCP}$ morphology is established during material synthesis; ordered morphologies including lamellae, hexagonally-packed cylinders, and spheres can be accessed based on the relative volume fractions $\left(f_{i}\right)$ and segregation strength $(\chi N)$ of the blocks. The translation of ordered bulk morphologies to thin films imposes significant perturbations to the free energy of the system due to the addition of interfaces with relatively large area. $^{2}$ BCP domain orientation is extremely sensitive to the nature of the interfacial interactions between each block and each interface. For most applications, a perpendicular BCP orientation is highly preferable, which necessitates balanced block-interface interactions at each interface. Directed self-assembly (DSA) has been established as an effective method for inducing alignment of $\mathrm{BCP}$ domains within the plane of the film once orientation control has been established.

Silicon-containing block copolymers (SiBCPs) are especially attractive as lithographic patterning materials because of the inherent oxygen plasma etch resistance of the Si block. ${ }^{3}$ However, control of feature orientation is rather challenging. The highly preferential interaction of the silicon-containing block with the top interface (air or vacuum) biases a parallel orientation of domains at all film thicknesses. The presence of only a neutral substrate surface is not sufficient to enable a perpendicular orientation of BCP domains. Annealing strategies that mitigate preferential top interface interactions have been developed that utilize solvent vapor, which both plasticizes the $\mathrm{BCP}$ to induce self-assembly and creates nonpreferential top interface interactions. ${ }^{4}$ We view traditional thermal annealing as a highly preferable process because it is both compatible with technology widely utilized in industry today ${ }^{5,6}$ and accesses thermodynamically minimum energy states. Control of the top interface is thus crucial to translate current thermal annealing processes to Si-BCPs.

\section{Experimental}

We have recently introduced polarityswitching top coats as a method of controlling the interactions between a BCP and the top interface. ${ }^{2}$ The general process is shown in Figure 1. A substrate surface is first applied and rendered insoluble. BCP is subsequently spin-coated onto the surface treatment. A polarity-switching top coat in its more polar form is applied from aqueous base onto the BCP film, which does not damage the BCP. Subsequent thermal annealing affects a polarity switch in the top coat that decreases its polarity and renders it a neutral surface for the BCP. Simultaneously, the BCP also selfassembles and orients perpendicular to the substrate. After thermal annealing, the top coat can be stripped with aqueous base and the BCP features can be etched. 


\section{Spin-coat and cross-link surface treatment}

2. Spin-coat block polymer

\section{Spin-coat topcoat}

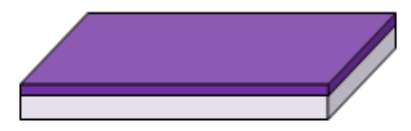

\section{Thermally anneal} + change polarity

\section{Strip topcoat}

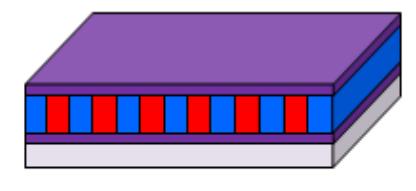

6. Etch
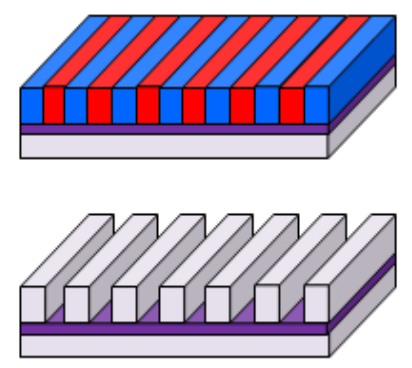

Figure 1. General top coat thermal annealing process.

\section{Results and discussion}

The polarity-switching top coat strategy should be applicable to a wide-variety of BCP materials. Herein we report the efficacy of the process with the Si-BCP poly(styrene-blocktrimethylsilylstyrene-block-styrene)

PS- $b$-PTMSS- $b$-PS annealed in the presence of top coat and substrate surface treatment can be oriented perpendicular to the substrate as shown in Figure 2. Herein we investigate the role of the top coat structure and interplay between each interface on the orientation of PS-b-PTMSS-b-PS

\section{Conclusions}

Top coats are effective at controlling the

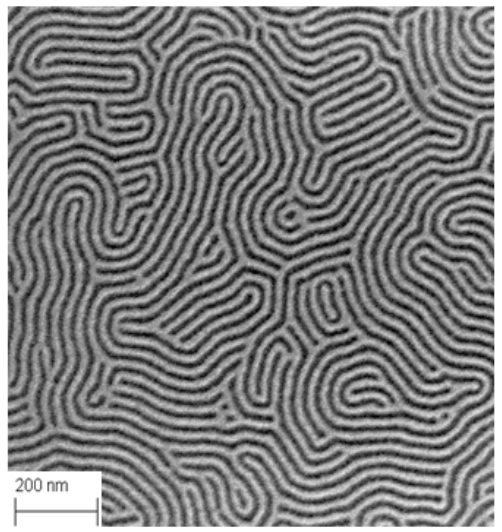

Figure 2. PS- $b$-PTMSS- $b$-PS annealed in the presence of a top coat produces a perpendicular orientation of BCP domains.

orientation of PS-b-PTMSS-b-PS. The role of various processing conditions is under investigation to maximize the efficacy of the top coat process.

\section{References:}

(1) Bates, F. S.; Fredrickson, G. H. Annual Review of Physical Chemistry 1990, 41, 525.

(2) Bates, C. M.; Seshimo, T.; Maher, M. J.; Durand, W. J.; Cushen, J. D.; Dean, L. M.; Blachut, G.; Ellison, C. J.; Willson, C. G. Science 2012, 338, 775.

(3) Ross, C. A.; Jung, Y. S.; Chuang, V. P.; Ilievski, F.; Yang, J. K. W.; Bita, I.; Thomas, E. L.; Smith, H. I.; Berggren, K. K.; Vancso, G. J.; Cheng, J. Y. J. Vac. Sci. Technol., B 2008, 26, 2489.

(4) Son, J. G.; Gotrik, K. W.; Ross, C. A. ACS Macro Letters 2012, 1279.

(5) Bencher, C.; Yi, H.; Zhou, J.; Cai, M.; Smith, J.; Miao, L.; Montal, O.; Blitshtein, S.; Lavi, A.; Dotan, K.; Dai, H.; Cheng, J. Y.; Sanders, D. P.; Tjio, M.; Holmes, S.; William, M. T., Ed.; SPIE: 2012; Vol. 8323, p 83230N.

(6) Liu, C.-C.; Ramirez-Hernandez, A.; Han, E.; Craig, G. S. W.; Tada, Y.; Yoshida, H.; Kang, H.; Ji, S.; Gopalan, P.; de, P. J. J.; Nealey, P. F. Macromolecules (Washington, DC, U. S.) 2013, 46, 1415. 